\title{
Tunable flexibility and porosity of the metal-organic framework DUT-49 through post-synthetic metal exchange
}

Bikash Garai, Volodymyr Bon, ${ }^{a}$ Simon Krause, ${ }^{a}$ Friedrich Schwotzer, Martin Gerlach, Irena Senkovska, ${ }^{\text {a }}$ Stefan Kaskel ${ }^{\mathrm{a}}$

a Anorganische Chemie I, Technische Universität Dresden, Bergstraße-66, 01069 Dresden, Germany

${ }^{\mathrm{b}}$ Macromolecular Crystallography Group, Helmholtz-Zentrum Berlin für Materialien und Energie, Albert-Einstein-Straße 15, 12489 Berlin, Germany

\section{Table of content}

Section

S1: Materials and methods

S2: Synthesis of the linker

S3: Experimental details for metal exchange

S4: Competitive metal-exchange

S5: Single crystal X-ray diffraction

S6: Powder X-ray diffraction

S7: Porosity and negative gas adsorption of heterometallic DUT-49

S8: Scanning electron microscopy and elemental mapping

S9: References
Page No.

S2

S3

S5

S6

S8

S11

S14

S16

S17 


\section{S1: Materials and methods}

All chemicals were purchased from available supplier and used after proper purification, if necessary. Carabzole, $N$-bromosuccinimide (NBS), potassium carbonate, copper (I) iodide, L-proline were purchased from Sigma Aldrich while 4,4'-diiodobiphenyl was procured from Alfa-Aesar. Solvents were purchased from local supplier and THF was purified from a solvent purification system. NMR spectra were measured with a BRUKER Avance $300 \mathrm{MHz}$ WBspectrometer. FTIR spectra were recorded with a Bruker spectrometer. Powder X-ray diffraction (PXRD) patterns were collected in transmission geometry with a STOE STADI P diffractometer operated at $40 \mathrm{kV}$ and $30 \mathrm{~mA}$ with monochromatic $\mathrm{Cu}-\mathrm{K} \alpha 1(\lambda=0.15405 \mathrm{~nm})$ radiation. Supercritical carbon dioxide activation for the samples was performed in a Jumbo Critical Point Dryer 13200J AB (SPI Supplies). The samples were washed thoroughly with NMP and then incubated in dry acetone. Acetone was exchanged for 10 times over a period of 5 days. The samples were then put into a sintered crucible, dipped in fresh acetone and are placed inside the dryer. Acetone was then exchanged with liquid carbon dioxide (purity: $99.995 \%$ ) at $17{ }^{\circ} \mathrm{C}$ for 10 times over a period of 5 days. After that, the temperature and pressure was raised beyond the critical point of $\mathrm{CO}_{2}$. The samples were allowed to remain in the supercritical fluid for around $15 \mathrm{~min}$ and then the supercritical fluid was released slowly within a time frame of $3 \mathrm{~h}$. The dried samples were flushed with Ar and then immediately transferred to an argonfilled glove box. $\mathrm{N}_{2}$ physisorption isotherms were measured at $77 \mathrm{~K}$ with a volumetric BELSORP-max device. The samples were degassed under dynamic vacuum at $100{ }^{\circ} \mathrm{C}$ prior to the measurement. SEM images of DUT-49 samples (prepared in argon) were taken with secondary electrons in a HITACHI SU8020 microscope using $1.0 \mathrm{kV}$ acceleration voltage. 


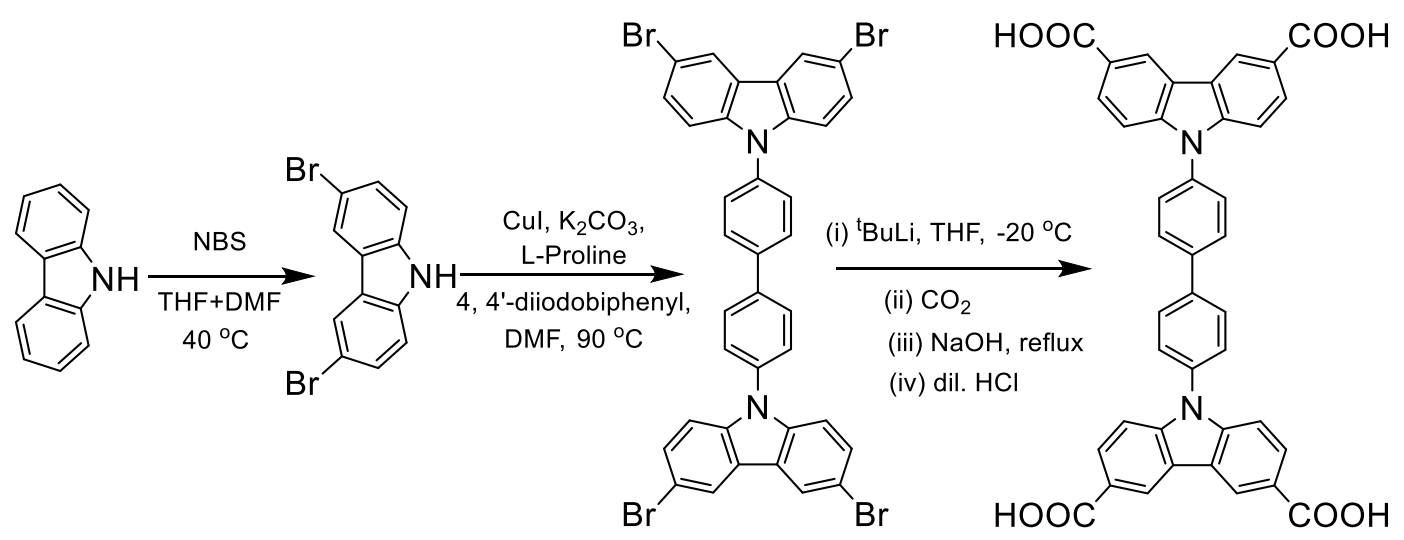

S cheme S1: Synthetic route for 9,9'-([1,1'-biphenyl]-4,4'-diyl)bis(9H-carbazole-3,6-dicarboxy lic acid) $\left(\mathrm{H}_{4} \mathrm{BBCDC}\right)$ linker.

The linker was synthesized using a minor modification of the previously reported procedures from our group. ${ }^{1,2}$ Carbazole (95\%, Sigma Aldrich) was first recrystallized from a 1:1 volumetric mixture of toluene and $\mathrm{EtOH}$ to obtain shiny white crystals. This recrystallized carbazole $(20.00 \mathrm{~g}$, $119.61 \mathrm{mmol}$ ) was dissolved in $300 \mathrm{ml}$ tetrahydrofuran (THF) and stirred at $40{ }^{\circ} \mathrm{C}$ for $15 \mathrm{~min} .50 .00 \mathrm{~g}$ (280.92mmol) of $N$-bromosuccinimide (NBS), dissolved in a solution of $200 \mathrm{ml}$ THF and $100 \mathrm{ml}$ $\mathrm{N}, \mathrm{N}$-dimethylformamide (DMF) was added slowly at $40{ }^{\circ} \mathrm{C}$ through a dropping funnel. The reaction mixture was then further stirred for additional $10 \mathrm{~h}$ at $40{ }^{\circ} \mathrm{C}$. THF fraction of the solvent was removed under reduced pressure and the remaining liquid was poured into ice water. The obtained precipitate was collected through filtration, dried and recrystallized from a mixture of dichloromethane and ethyl acetate to get $30.00 \mathrm{~g}$ of pure 3,6-dibromocarbazole as the product. ${ }^{1} \mathrm{H}$ NMR (300 MHz, THF-d ${ }^{8}$ ), ppm: 10.59(s, 1H), 8.26(s, 2H), 7.46(m, 2H), 7.33(m, 2H).

3,6-dibromocarbazole $(2.40 \mathrm{~g}, 7.39 \mathrm{mmol})$ was taken in a Schlenk flask along with 4,4'diiodobiphenyl (1.00 g, $2.46 \mathrm{mmol})$, CuI (117 mg, $0.61 \mathrm{mmol}), L$-Proline (142 mg, $1.23 \mathrm{mmol})$ and $\mathrm{K}_{2} \mathrm{CO}_{3}(5.45 \mathrm{~g}, 39.41 \mathrm{mmol})$ under Ar atmosphere. Then $60 \mathrm{ml}$ degassed DMF was added to the mixture and heated to $90{ }^{\circ} \mathrm{C}$ for 5 days. The reaction mixture was then poured into water and the ppt. was filtered, washed with EtOH, ethyl acetate (EtOAc) and dichloromethane (DCM) to get $1.45 \mathrm{~g}$ of the coupled product as an insoluble mass. FTIR (ATR, $\mathrm{cm}^{-1}$ ): $1853(\mathrm{w}), 1678(\mathrm{w}), 1601$ (w), $1504(\mathrm{~s})$, 1467 (s), 1436 (s), 1365 (m), 1317 (m), 1278 (s), 1228 (s), 1176 (w), 1118 (w), 1053 (m), 1020 (m), 939 (m), 866 (s), $814(\mathrm{~s}), 794$ (s), $723(\mathrm{~m}), 665$ (w), $634(\mathrm{~m})$.

$2.85 \mathrm{~g}(3.56 \mathrm{mmol})$ of the tetra-bromo product was suspended into $400 \mathrm{ml}$ dry THF taken in a round bottom flask. The suspension was cooled to $-15{ }^{\circ} \mathrm{C}$ and then $17.5 \mathrm{ml}$ of ${ }^{t} \mathrm{BuLi}(1.7 \mathrm{M}$ in pentane) was added, while maintaining the temperature. The temperature was then slowly increased to $0{ }^{\circ} \mathrm{C}$ over a period of $3 \mathrm{~h}$ and $4.5 \mathrm{ml}$ of ${ }^{t} \mathrm{BuLi}$ solution was added. The suspension was stirred for another two hours and then another additional $4.5 \mathrm{ml}$ of ${ }^{t} \mathrm{BuLi}$ solution was added. The solution was then stirred for another $30 \mathrm{~min}$ and then dry gaseous $\mathrm{CO}_{2}$ was bubbled through the reaction mixture for a period of $5 \mathrm{~min}$. The resulting precipitate was then filtered and washed with water. The crude product was then refluxed with $300 \mathrm{ml} \mathrm{NaOH}$ solution and any insoluble residue was filtered off. The filtrate was then acidified with dilute $\mathrm{HCl}$ to $\mathrm{pH}=2$ and then the precipitate was filtered and washed with water till the wash liquid became neutral. The obtained product was then further recrystallized 
from $\mathrm{N}$-methyl-2-pyrrolidon (NMP) to obtain $1.50 \mathrm{~g}$ of the linker. ${ }^{1} \mathrm{H}$ NMR (200 MHz, DMSO-d $\mathrm{d}^{6}$ ), ppm: 12.85 (s, 4H), $9.02(\mathrm{~s}, 4 \mathrm{H}), 8.16(\mathrm{~m}, 8 \mathrm{H}), 7.89(\mathrm{~m}, 4 \mathrm{H}), 7.58(\mathrm{~m}, 4 \mathrm{H})$. 


\section{S3: Experimental de tails for me tal exchange}

Table S1: Details of the metal conc. and time required for complete exchange to DUT-49(Co)

\begin{tabular}{|c|c|c|}
\hline Metal salt & Conc. in NMP solution & Incubation time \\
\hline $\mathrm{Mn}\left(\mathrm{NO}_{3}\right)_{2} \cdot 6 \mathrm{H}_{2} \mathrm{O}$ & $0.1 \mathrm{M}$ & $24 \mathrm{~h}$ \\
\hline $\mathrm{FeSO}_{4} .7 \mathrm{H}_{2} \mathrm{O}$ & Saturated solution $(<0.1 \mathrm{M})$ & $24 \mathrm{~h}$ \\
\hline $\mathrm{Ni}\left(\mathrm{NO}_{3}\right)_{2} \cdot 6 \mathrm{H}_{2} \mathrm{O}$ & $0.2 \mathrm{M}$ & $24 \mathrm{~h}$ \\
\hline $\mathrm{Cu}\left(\mathrm{NO}_{3}\right)_{2} \cdot 3 \mathrm{H}_{2} \mathrm{O}$ & $0.1 \mathrm{M}$ & $3 \mathrm{~h}$ \\
\hline $\mathrm{Zn}\left(\mathrm{NO}_{3}\right)_{2} \cdot 6 \mathrm{H}_{2} \mathrm{O}$ & $0.1 \mathrm{M}$ & $24 \mathrm{~h}$ \\
\hline $\mathrm{Cd}\left(\mathrm{NO}_{3}\right)_{2} \cdot 6 \mathrm{H}_{2} \mathrm{O}$ & $0.2 \mathrm{M}$ & $72 \mathrm{~h}$ \\
\hline
\end{tabular}

For the partial exchange with $\mathrm{Cu}(\mathrm{II})$, a measured amount (volume measured while in solution, then metal content was estimated considering the density and structural formula) of the MOF crystals was taken into a glass vial and then added with fresh NMP on the top of the crystals $(\sim 3 \mathrm{~cm}$ height of the solvent column). The crystals were allowed to settle down for next $30 \mathrm{~min}$, and thereafter a measured amount of the $\mathrm{Cu}\left(\mathrm{NO}_{3}\right)_{2} \cdot 3 \mathrm{H}_{2} \mathrm{O}$ solution (conc. $0.01 \mathrm{M}$ ) in NMP was added slowly on top of the solvent. The solution was slowly diffused to reach the crystals at the bottom of the vial and after that the vial was stirred occasionally to maximize the contact of the crystals with the metal ion solution. After $4 \mathrm{~h}$, the solution was replaced with fresh NMP and the crystals were washed with fresh NMP for at least 5 times before further usage.

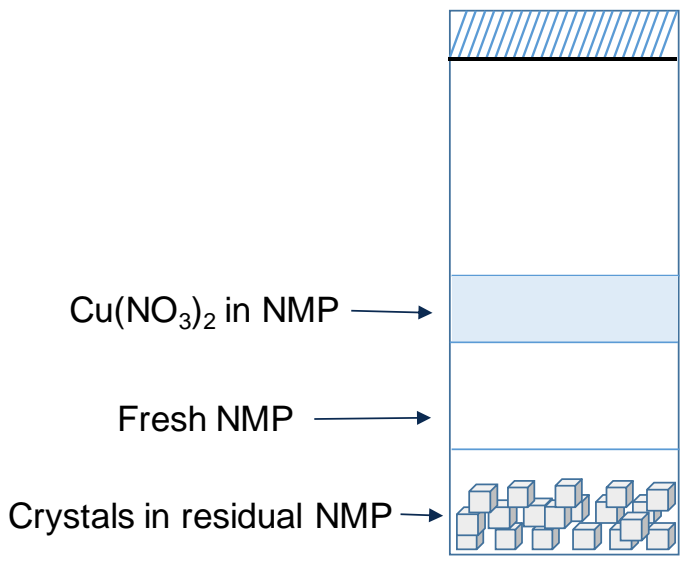

Figure S1: Representation for the lay ered mixture of solvent and solution, used for heterometallic DUT-49 synthesis. 


\section{S4: Competitive metal exchange}

For studying the competitive the behavior of different metal ions towards substitution from DUT-49 framework, first DUT-49(Co) crystal was exchanged with 0.1 M NMP solution of all the metal ions. After the incubation period of $12 \mathrm{~h}$, the crystals were washed with fresh NMP over 3 days until the solution became colorless. These obtained crystals were then separately subjected to another round of exchange with the rest of metal ions from a 0.1 M NMP solution. These final crystals were then washed with NMP ( 3 days until the clear solution), followed by acetone ( 5 times in 2 days) and then dried at $80{ }^{\circ} \mathrm{C}$ oven before analyzing the metal content with ICP-OES analysis. For ICP-OES, a measured amount of the MOF crystal was digested with $1 \mathrm{ml}$ freshly prepared aqua regia and then sonicated for $10 \mathrm{~min}$. The solution was then diluted to $15 \mathrm{ml}$ with deionized water and analyzed for metal content. Based on the conc. of the solution, some solutions were further diluted to fit within the analysis range of the device.

Table-S 2: Details of the metal ion conc. obtained from ICP-OES for the competitive metal exchange study

\begin{tabular}{|c|c|c|c|c|}
\hline & $1^{\text {st }}$ exchange & \multicolumn{3}{|l|}{$2^{\text {110 }}$ exchange } \\
\hline \multirow{21}{*}{$\begin{array}{l}\text { DUT-49(Co) } \\
\text { Mn: } 0 \% \text {, Fe: } \\
0 \%, \text { Co: } 100 \% \text {, } \\
\text { Ni: } 0 \%, \mathrm{Cu}: \\
0 \%, \mathrm{Zn}: 0 \% \text {, } \\
\text { Cd: } 0 \%\end{array}$} & \multirow{5}{*}{$\begin{array}{l}\text { Mn(II) } \\
\text { Mn: 55\%, Fe: } 0 \%, \text { Co: } \\
45 \% \text {, Ni: } 0 \%, \mathrm{Cu}: 0 \%, \mathrm{Zn}: \\
0 \%, \mathrm{Cd}: 0 \%\end{array}$} & $\mathrm{Fe}(\mathrm{II})$ & $\mathrm{Co}(\mathrm{II})$ & $\mathrm{Ni}(\mathrm{II})$ \\
\hline & & Mn: $8 \%$, Fe: $83 \%$, Co: & $\mathrm{Mn}: 1 \%$, Fe: $0 \%$, Co: & $\mathrm{Mn}: 3 \%$, Fe: $0 \%$, Co: \\
\hline & & $\begin{array}{l}9 \%, \mathrm{Ni}: 0 \%, \mathrm{Cu}: 0 \%, \\
\text { Zn: } 0 \%, \mathrm{Cd}: 0 \%\end{array}$ & $\begin{array}{l}100 \%, \text { Ni: } 0 \%, \mathrm{Cu}: \\
0 \% \text {, Zn: } 0 \%, \text { Cd: } 0 \%\end{array}$ & $\begin{array}{l}13 \%, \mathrm{Ni}: 84 \%, \mathrm{Cu}: \\
0 \%, \mathrm{Zn}: 0 \%, \mathrm{Cd}: 0 \%\end{array}$ \\
\hline & & $\mathrm{Cu}(\mathrm{II})$ & $\mathrm{Zn}(\mathrm{II})$ & $\mathrm{Cd}(\mathrm{II})$ \\
\hline & & $\begin{array}{l}\text { Mn: } 0 \%, \text { Fe: } 0 \%, \mathrm{Co:} \\
0 \% \text {, Ni: } 0 \%, \mathrm{Cu}: 100 \% \text {, } \\
\text { Zn: } 0 \%, \mathrm{Cd}: 0 \%\end{array}$ & $\begin{array}{l}\text { Mn: } 0 \%, \text { Fe: } 0 \%, \text { Co: } \\
0 \% \text {, Ni: } 0 \% \text {, Cu: } 0 \% \text {, } \\
\text { Zn: } 100 \%, \text { Cd: } 0 \%\end{array}$ & $\begin{array}{l}\mathrm{Mn}: 3 \%, \mathrm{Fe}: 0 \%, \mathrm{Co}: \\
0 \% \text {, Ni: } 0 \%, \mathrm{Cu}: 0 \% \text {, } \\
\text { Zn: } 0 \%, \mathrm{Cd}: 97 \%\end{array}$ \\
\hline & \multirow{4}{*}{$\begin{array}{l}\mathrm{Fe}(\mathrm{II}) \\
\text { Mn: 0\%, Fe: 65\%, Co: } \\
35 \% \text {, Ni: } 0 \%, \mathrm{Cu}: 0 \%, \mathrm{Zn}: \\
0 \%, \mathrm{Cd}: 0 \%\end{array}$} & $\mathrm{Mn}(\mathrm{II})$ & $\mathrm{Co}(\mathrm{II})$ & $\mathrm{Ni}(\mathrm{II})$ \\
\hline & & $\begin{array}{l}\mathrm{Mn}: 37 \%, \mathrm{Fe}: 57 \% \text {, } \\
\mathrm{Co}: 6 \%, \mathrm{Ni}: 0 \%, \mathrm{Cu}: \\
0 \%, \mathrm{Zn}: 0 \%, \mathrm{Cd}: 0 \%\end{array}$ & $\begin{array}{l}\text { Mn: } 0 \% \text {, Fe: } 66 \% \text {, Co: } \\
34 \% \text {, Ni: } 0 \%, \mathrm{Cu}: 0 \% \text {, } \\
\text { Zn: } 0 \%, \mathrm{Cd}: 0 \%\end{array}$ & $\begin{array}{l}\text { Mn: } 0 \%, \mathrm{Fe}: 58 \%, \mathrm{Co}: \\
\text { 2\%, Ni: } 40 \%, \mathrm{Cu}: 0 \% \text {, } \\
\text { Zn: } 0 \%, \mathrm{Cd}: 0 \%\end{array}$ \\
\hline & & $\mathrm{Cu}(\mathrm{II})$ & $\mathrm{Zn}(\mathrm{II})$ & $\mathrm{Cd}(\mathrm{II})$ \\
\hline & & $\begin{array}{l}\mathrm{Mn}: 0 \%, \mathrm{Fe}: 55 \%, \mathrm{Co}: \\
45 \% \text {, Ni: } 0 \%, \mathrm{Cu}: 0 \% \text {, } \\
\mathrm{Zn}: 0 \%, \mathrm{Cd}: 0 \%\end{array}$ & $\begin{array}{l}\text { Mn: } 0 \%, \mathrm{Fe}: 62 \%, \mathrm{Co}: \\
0 \% \text {, Ni: } 0 \%, \mathrm{Cu}: 0 \% \text {, } \\
\mathrm{Zn:} 38 \%, \mathrm{Cd}: 0 \%\end{array}$ & $\begin{array}{l}\text { Mn: } 0 \% \text {, Fe: } 49 \% \text {, Co: } \\
2 \% \text {, Ni: } 0 \%, \mathrm{Cu}: 0 \% \text {, } \\
\text { Zn: } 0 \%, \mathrm{Cd}: 49 \%\end{array}$ \\
\hline & \multirow{4}{*}{$\begin{array}{l}\text { Ni(II) } \\
\text { Mn: } 0 \% \text {, Fe: } 0 \%, \text { Co: } 56 \% \text {, } \\
\text { Ni: } 44 \%, \mathrm{Cu}: 0 \%, \mathrm{Zn}: 0 \% \text {, } \\
\text { Cd: } 0 \%\end{array}$} & $\mathrm{Mn}(\mathrm{II})$ & $\mathrm{Fe}(\mathrm{II})$ & $\operatorname{Co}(\mathrm{II})$ \\
\hline & & $\begin{array}{l}\text { Mn: } 100 \%, \text { Fe: } 0 \% \text {, } \\
\text { Co: } 0 \%, \text { Ni: } 0 \%, \mathrm{Cu}: \\
0 \%, \mathrm{Zn}: 0 \%, \mathrm{Cd}: 0 \%\end{array}$ & $\begin{array}{l}\text { Mn: } 0 \% \text {, Fe: } 53 \%, \text { Co: } \\
22 \%, \quad \text { Ni: } 25 \%, \quad \mathrm{Cu}: \\
0 \%, \mathrm{Zn}: 0 \%, \mathrm{Cd}: 0 \%\end{array}$ & $\begin{array}{l}\text { Mn: 0\%, Fe: } 0 \%, \text { Co: } \\
\text { 94\%, Ni: } 6 \%, \mathrm{Cu}: 0 \% \text {, } \\
\text { Zn: } 0 \%, \mathrm{Cd}: 0 \%\end{array}$ \\
\hline & & $\mathrm{Cu}(\mathrm{II})$ & $\mathrm{Zn}(\mathrm{II})$ & $\mathrm{Cd}(\mathrm{II})$ \\
\hline & & $\begin{array}{l}\text { Mn: } 0 \%, \mathrm{Fe}: 0 \%, \mathrm{Co}: \\
0 \% \text {, Ni: } 0 \%, \mathrm{Cu}: 100 \% \text {, } \\
\text { Zn: } 0 \%, \mathrm{Cd}: 0 \%\end{array}$ & $\begin{array}{l}\text { Mn: } 0 \%, \text { Fe: } 0 \%, \mathrm{Co}: \\
0 \%, \mathrm{Ni}: 0 \%, \mathrm{Cu}: 0 \% \text {, } \\
\text { Zn: } 100 \%, \mathrm{Cd}: 0 \%\end{array}$ & $\begin{array}{l}\mathrm{Mn}: 0 \%, \mathrm{Fe}: 0 \%, \mathrm{Co}: \\
\text { 3\%, Ni: } 0 \%, \mathrm{Cu}: 0 \% \text {, } \\
\text { Zn: } 0 \%, \mathrm{Cd}: 97 \%\end{array}$ \\
\hline & \multirow{4}{*}{$\begin{array}{l}\mathrm{Cu}(\mathrm{II}) \\
\text { Mn: } 0 \%, \mathrm{Fe}: 0 \%, \mathrm{Co}: 0 \% \text {, } \\
\text { Ni: } 0 \%, \mathrm{Cu}: 100 \%, \mathrm{Zn}: 0 \% \text {, } \\
\text { Cd: } 0 \%\end{array}$} & $\mathrm{Mn}(\mathrm{II})$ & $\mathrm{Fe}(\mathrm{II})$ & $\mathrm{Co}(\mathrm{II})$ \\
\hline & & $\begin{array}{l}\text { Mn: } 0 \% \text {, Fe: } 0 \%, \mathrm{Co}: \\
0 \% \text {, Ni: } 0 \%, \mathrm{Cu}: 100 \% \text {, } \\
\text { Zn: } 0 \%, \mathrm{Cd}: 0 \%\end{array}$ & $\begin{array}{l}\text { Mn: } 0 \%, \mathrm{Fe}: 30 \%, \mathrm{Co}: \\
0 \%, \mathrm{Ni}: 0 \%, \mathrm{Cu}: 70 \% \text {, } \\
\mathrm{Zn}: 0 \%, \mathrm{Cd}: 0 \%\end{array}$ & $\begin{array}{l}\text { Mn: } 0 \%, \text { Fe: } 0 \%, \mathrm{Co}: \\
0 \%, \quad \mathrm{Ni}: 0 \%, \mathrm{Cu}: \\
100 \%, \mathrm{Zn}: 0 \%, \mathrm{Cd}: \\
0 \%\end{array}$ \\
\hline & & $\mathrm{Ni}(\mathrm{II})$ & $\mathrm{Zn}(\mathrm{II})$ & $\mathrm{Cd}(\mathrm{II})$ \\
\hline & & $\begin{array}{l}\text { Mn: } 0 \% \text {, Fe: } 0 \%, \mathrm{Co}: \\
0 \% \text {, Ni: } 0 \%, \mathrm{Cu}: 100 \% \text {, } \\
\text { Zn: } 0 \%, \mathrm{Cd}: 0 \%\end{array}$ & $\begin{array}{l}\text { Mn: } 0 \%, \text { Fe: } 0 \%, \text { Co: } \\
0 \%, \quad \mathrm{Ni}: 0 \%, \quad \mathrm{Cu}: \\
100 \%, \mathrm{Zn}: 0 \%, \mathrm{Cd}: \\
0 \%\end{array}$ & $\begin{array}{l}\text { Mn: } 0 \%, \text { Fe: } 0 \%, \mathrm{Co}: \\
0 \%, \quad \mathrm{Ni}: 0 \%, \mathrm{Cu}: \\
100 \%, \mathrm{Zn}: 0 \%, \mathrm{Cd}: \\
0 \%\end{array}$ \\
\hline & \multirow{4}{*}{$\begin{array}{l}\mathrm{Zn}(\mathrm{II}) \\
\text { Mn: } 0 \%, \mathrm{Fe}: 0 \%, \mathrm{Co}: 0 \% \text {, } \\
\text { Ni: } 0 \%, \mathrm{Cu}: 0 \%, \mathrm{Zn}: 100 \% \text {, } \\
\text { Cd: } 0 \%\end{array}$} & $\mathrm{Mn}(\mathrm{II})$ & $\mathrm{Fe}(\mathrm{II})$ & $\mathrm{Co}(\mathrm{II})$ \\
\hline & & $\begin{array}{l}\mathrm{Mn}: 0 \%, \mathrm{Fe}: 0 \%, \mathrm{Co}: \\
0 \%, \mathrm{Ni}: 0 \%, \mathrm{Cu}: 0 \% \text {, } \\
\text { Zn: } 100 \%, \mathrm{Cd}: 0 \%\end{array}$ & $\begin{array}{l}\text { Mn: } 0 \% \text {, Fe: } 40 \% \text {, Co: } \\
0 \% \text {, Ni: } 0 \% \text {, Cu: } 0 \% \text {, } \\
\text { Zn: } 60 \% \text {, Cd: } 0 \%\end{array}$ & $\begin{array}{l}\text { Mn: } 0 \% \text {, Fe: } 0 \% \text {, Co: } \\
31 \% \text {, Ni: } 0 \% \text {, Cu: } 0 \% \text {, } \\
\text { Zn: } 69 \% \text {, Cd: } 0 \%\end{array}$ \\
\hline & & $\mathrm{Ni}(\mathrm{II})$ & $\mathrm{Cu}(\mathrm{II})$ & $\mathrm{Cd}(\mathrm{II})$ \\
\hline & & $\begin{array}{l}\text { Mn: } 0 \%, \text { Fe: } 0 \% \text {, Co: } \\
0 \% \text {, Ni: } 10 \% \text {, Cu: } 0 \% \text {, } \\
\text { Zn: } 90 \%, \mathrm{Cd}: 0 \%\end{array}$ & $\begin{array}{l}\text { Mn: } 0 \%, \text { Fe: } 0 \%, \text { Co: } \\
0 \%, \quad \text { Ni: } 0 \%, \quad \text { Cu: } \\
100 \%, \quad \mathrm{Zn}: 0 \%, \text { Cd: }\end{array}$ & $\begin{array}{l}\text { Mn: } 0 \%, \text { Fe: } 0 \%, \mathrm{Co:} \\
0 \%, \mathrm{Ni}: 0 \%, \mathrm{Cu}: 0 \% \text {, } \\
\text { Zn: } 67 \%, \mathrm{Cd}: 33 \%\end{array}$ \\
\hline
\end{tabular}




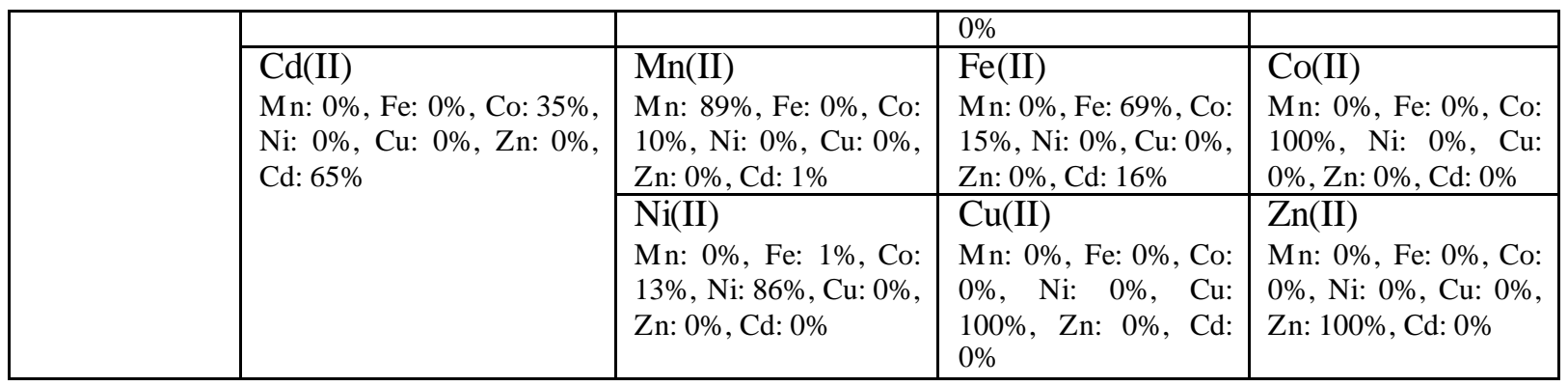

Table-S3: ICP-OES measured metal ion conc. in DUT-49(M) MOFs, obtained from complete metal exchange (total incubation as mentioned in table S1) to DUT-49(Co)

\begin{tabular}{|c|c|}
\hline MOF & Relative abundance of different metal ions \\
\hline$\overline{\text { DUT-49(Mn) }}$ & Mn: $99.9 \%$, Fe: $0 \%$, Co: $0.1 \%$, Ni: $0 \%$, Cu: $0 \%, \mathrm{Zn}: 0 \%$, Cd: $0 \%$ \\
\hline DUT-49(Fe) & Mn: $0 \%$, Fe: $98 \%$, Co: $2 \%$, Ni: $0 \%$, Cu: 0\%, Zn: 0\%, Cd: $0 \%$ \\
\hline DUT-49(Co) & Mn: $0 \%$, Fe: $0 \%$, Co: $100 \%$, Ni: $0 \%$, Cu: $0 \%, \mathrm{Zn}: 0 \%$, Cd: $0 \%$ \\
\hline DUT-49(Ni) & Mn: $0 \%$, Fe: $10 \%$, Co: $6 \%$, Ni: $84 \%$, Cu: $0 \%$, Zn: $0 \%$, Cd: $0 \%$ \\
\hline DUT-49(Cu) & Mn: $0 \%$, Fe: $0 \%$, Co: $0 \%$, Ni: 0\%, Cu: $100 \%$, Zn: $0 \%$, Cd: $0 \%$ \\
\hline DUT-49(Zn) & Mn: $0 \%$, Fe: $0 \%$, Co: $0 \%$, Ni: 0\%, Cu: 0\%, Zn: $100 \%$, Cd: $0 \%$ \\
\hline DUT-49(Cd) & Mn: $0 \%$, Fe: $0 \%$, Co: $0 \%$, Ni: $0 \%$, Cu: 0\%, Zn: 0\%, Cd: $100 \%$ \\
\hline
\end{tabular}




\section{S5: Single crystal $\mathrm{X}$-ray diffraction}

A suitably sized cubic crystal of the DUT-49(M) MOF from NMP was brought into a borosilicate glasscapillary $(d=0.3 \mathrm{~mm})$ with small amount of the mother liquor. The datasets were collected at BESSY MX BL14.3 beamline of Helmholtz-Zentrum Berlin für Materialien und Energie. ${ }^{3}$ Monochromatic X-ray radiation with a wavelength of $\lambda=0.089499 \mathrm{~nm}(E=13.85 \mathrm{keV})$ was used in experiments. All datasets were collected at room temperature. The crystal symmetry and scan range were determined in each particular case using iMosflmprogram. ${ }^{4,5}$ The $\varphi$-scans with oscillation range of $1^{\circ}$ were used for data collection. For each dataset, 60 images were collected to reach the maximal completeness. The datasets were processed in automatic regime using XDSAPP 2.0 software. ${ }^{6}$ Crystal structures were solved by direct methods and refined by full matrix least-squares on $F^{2}$ using SHELX2018/3 program package. ${ }^{7,8}$ All non-hydrogen atoms were refined in anisotropic approximation. Hydrogen atoms were refined in geometrically calculated positions using "riding model" with $\operatorname{Uiso}(H)=1.2 \operatorname{Uiso}(C)$. The large pores, high crystal symmetry and room temperature data collection lead to disorder of solvent molecules within the pores of the frameworks. SQUEEZE routine in PLATON was used to generate the reflection intensities with subtracted solvent contribution. ${ }^{9}$ CCDC1957602-1957605 contain the supplementary crystallographic data for DUT-49(Mn), DUT-49(Fe), DUT-49(Ni) and DUT-49(Cd) correspondingly. These data can be obtained free of charge from the Cambridge Crystallographic Data Centre via www.ccdc.cam.ac.uk/data_request/cif. Experimental data on single crystal X-ray experiments are summarized in table $\mathbf{S 4}$.

Table-S 4: Summary of single crystal X-ray diffraction data for DUT-49 MOFs

\begin{tabular}{|c|c|c|c|c|}
\hline & DUT-49(Mn) & DUT-49(Fe) & DUT-49(Ni) & DUT-49(Cd) \\
\hline Empirical formula & $\mathrm{C}_{40} \mathrm{H}_{20} \mathrm{Mn}_{2} \mathrm{~N}_{2} \mathrm{O}_{10}$ & $\mathrm{C}_{40} \mathrm{H}_{20} \mathrm{Fe}_{2} \mathrm{~N}_{2} \mathrm{O}_{10}$ & $\mathrm{C}_{40} \mathrm{H}_{20} \mathrm{Ni}_{2} \mathrm{~N}_{2} \mathrm{O}_{10}$ & $\mathrm{C}_{40} \mathrm{H}_{20} \mathrm{Cd}_{2} \mathrm{~N}_{2} \mathrm{O}_{10}$ \\
\hline Formula weight & 798.46 & 800.28 & 806.00 & 913.38 \\
\hline $\begin{array}{ll}\text { Measurement } & \text { Temp. } \\
(\mathrm{K}) & \end{array}$ & 296 & 296 & 296 & 296 \\
\hline Crystal system & Cubic & Cubic & Cubic & Cubic \\
\hline Space group & $F m \overline{3} m$ & $F m \overline{3} m$ & $F m \overline{3} m$ & $F m \overline{3} m$ \\
\hline Unit cell dimension $(\check{A})$ & $47.080(5)$ & $46.890(5)$ & $46.820(5)$ & $47.420(6)$ \\
\hline Unit cell volume $\left(\AA^{3}\right)$ & 104354(36) & $103096(36)$ & $102635(36)$ & $106631(37)$ \\
\hline $\mathrm{Z}$ & 24 & 24 & 24 & 24 \\
\hline $\begin{array}{l}\text { Calculated density } \\
\left(\mathrm{gcm}^{-3}\right)\end{array}$ & 0.305 & 0.309 & 0.313 & 0.341 \\
\hline$\mu, \mathrm{mm}^{-1}$ & 0.295 & 0.339 & 0.432 & 0.462 \\
\hline$F(000)$ & 9696 & 9744 & 9840 & 10800 \\
\hline$\theta$ range $\left(^{\circ}\right)$ & $1.09-31.75$ & $1.81-31.72$ & $1.09-31.36$ & $1.08-31.74$ \\
\hline Limiting indices & $\begin{array}{l}-44 \leq \mathrm{h} \leq 48 \\
-52 \leq k \leq 47 \\
-48 \leq l \leq 14\end{array}$ & $\begin{array}{l}-45 \leq h \leq 43 \\
-50 \leq k \leq 43 \\
-50 \leq l \leq 26\end{array}$ & $\begin{array}{l}-46 \leq h \leq 44 \\
-51 \leq k \leq 46 \\
-51 \leq l \leq 42\end{array}$ & $\begin{array}{l}-45 \leq h \leq 48 \\
-53 \leq k \leq 46 \\
-51 \leq l \leq 23\end{array}$ \\
\hline $\begin{array}{l}\text { Reflections } \\
\text { collected/Unique } \\
\text { reflection }\end{array}$ & $\begin{array}{l}37732 / 4167 \\
R_{\text {int }}=0.0218\end{array}$ & $\begin{array}{l}44879 / 3994 \\
R_{\text {int }}=0.0296\end{array}$ & $\begin{array}{l}69188 / 4007 \\
R_{\text {int }}=0.0461\end{array}$ & $\begin{array}{l}47440 / 4261 \\
R_{\text {int }}=0.0192\end{array}$ \\
\hline Data/parameters & $4197 / 97$ & $3994 / 97$ & $4007 / 97$ & $4261 / 89$ \\
\hline GooF on $F^{2}$ (all data) & 1.106 & 1.442 & 1.002 & 1.076 \\
\hline$R[\mathrm{I}>2 \sigma(\mathrm{I})]$ & 0.0619 & 0.0929 & 0.0911 & 0.0729 \\
\hline$w R 2$ (all data) & 0.2344 & 0.3261 & 0.3246 & 0.2862 \\
\hline $\begin{array}{l}\text { Largest diff. peak and } \\
\text { holes, } \mathrm{e} / \AA^{3}\end{array}$ & $0.630 /-0.371$ & $0.882 /-0.712$ & $1.237 /-0.477$ & $0.722 /-0.424$ \\
\hline CCDC number & 1957604 & 1957603 & 1957605 & 1957602 \\
\hline
\end{tabular}




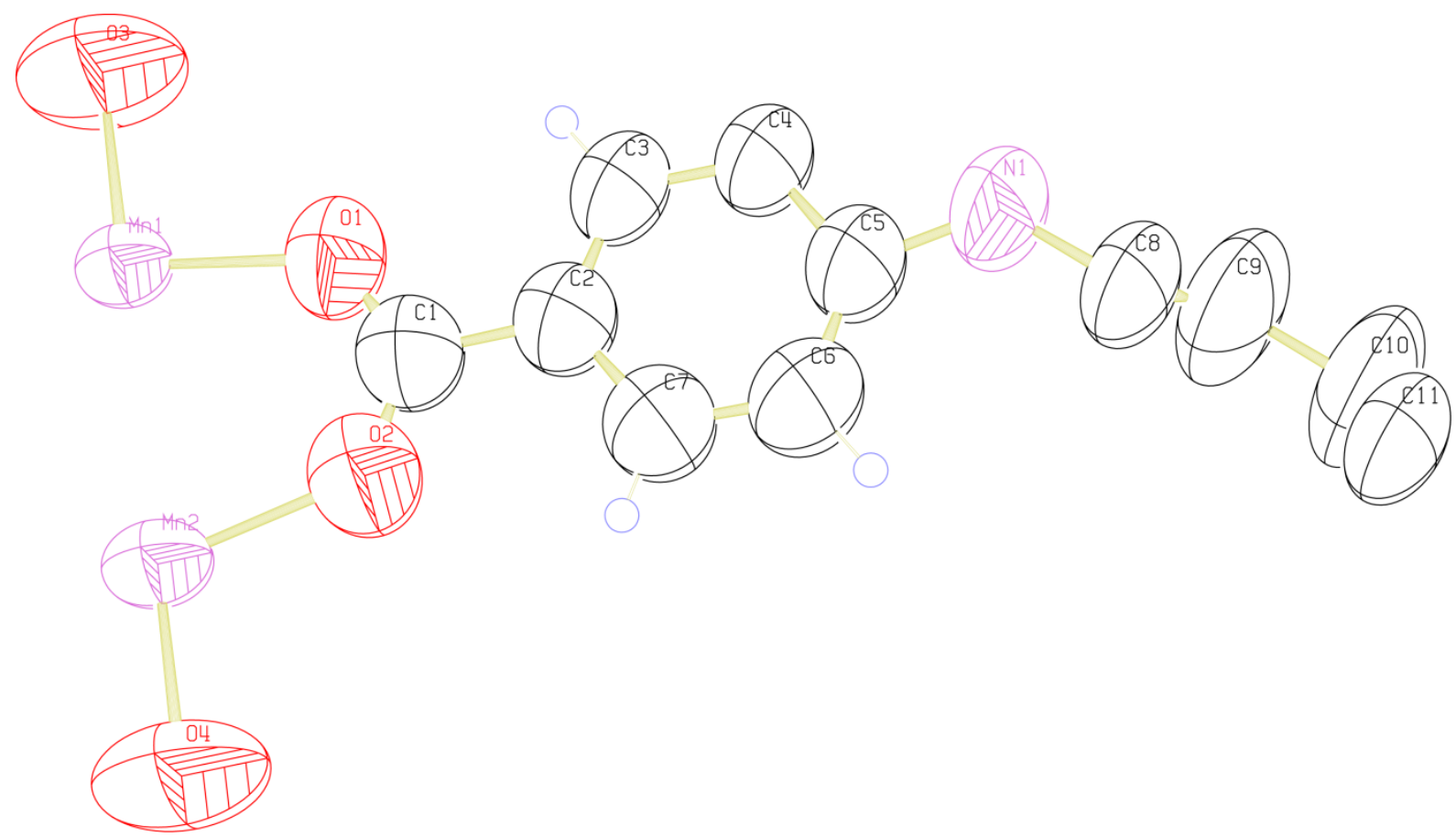

Figure S2: ORTEP diagram for asymmetric unit of DUT-49(Mn). The thermal ellipsoids are set at a 50\% probability level.

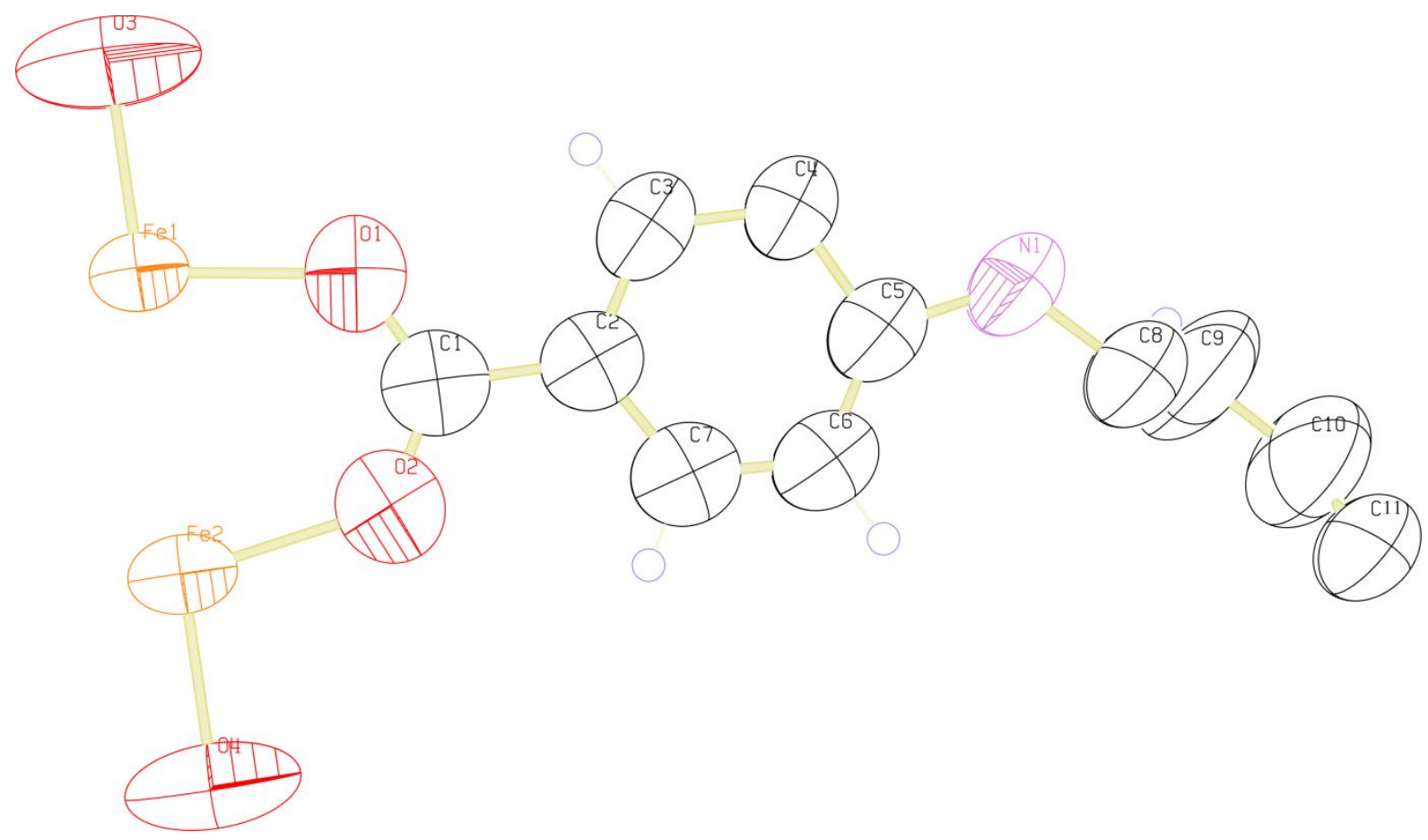

Figure S3: ORTEP diagram for asymmetric unit of DUT-49(Fe). The thermal ellipsoids are set at a 50\% probability level.

SP 


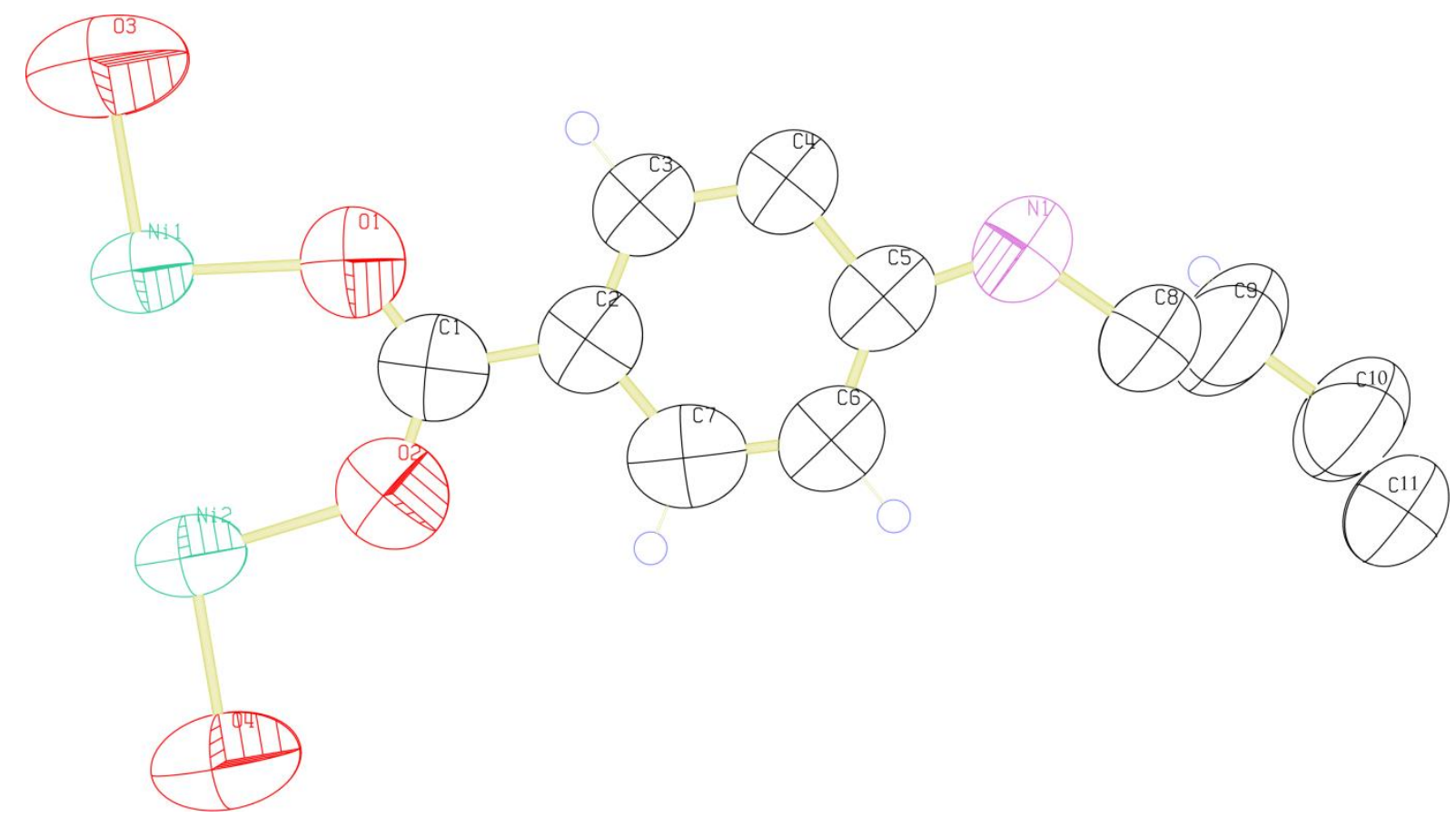

Figure S4: ORTEP diagram for asy mmetric unit of DUT-49(Ni). The thermal ellipsoids are set at a 50\% probability level.

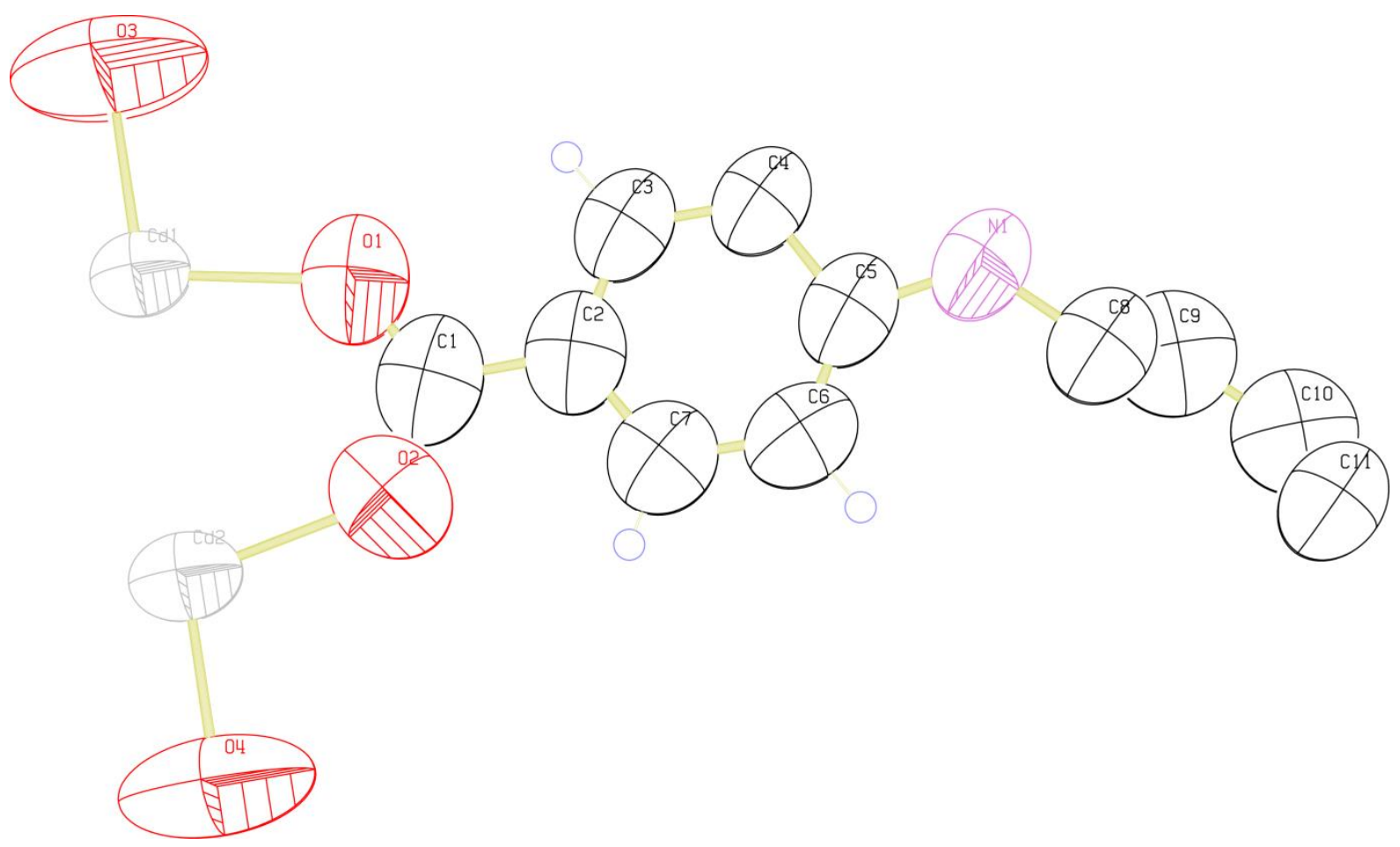

Figure S5: ORTEP diagram for asy mmetric unit of DUT-49(Cd). The thermal ellipsoids are set at a 50\% probability level. 


\section{S6: Powder X-ray diffraction}

The crystal samples of the DUT-49(M) were first put into a thin polymer foil and the residual solvent was soaked off from the powder. The sample was closed between two foils by vacuum grease to fix the samples within the film. This was then mounted on a STOE diffractometer and diffraction patterns were collected in transmittance mode. The peak like curves (at $2 \theta \sim 2.26^{\circ}$ ) in the green patterns of $\mathbf{S 6} \mathbf{a}, \mathbf{b}$ are instrumental artifacts.
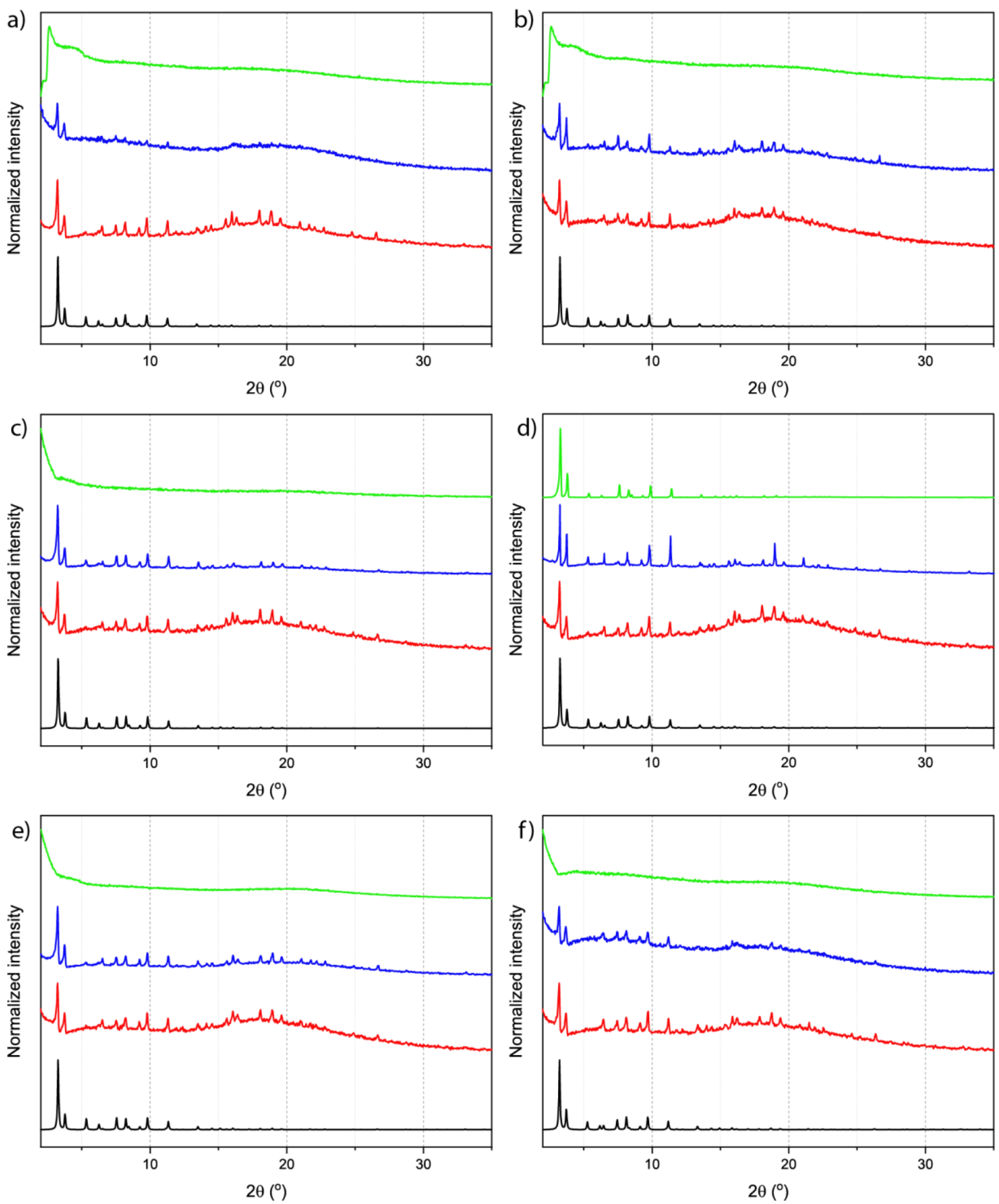

Figure S6: XRD patterns of a) DUT-49(Mn), b) DUT-49(Fe), c) DUT-49(Co), d) DUT-49(Ni), e) DUT-49(Zn) and f) DUT$49(\mathrm{Cd})$ MOF from NMP (red pattern), after acetone exchange (blue) and after supercritical $\mathrm{CO}_{2}$ activation (green), and comparison with respective calculated patterns (black). 


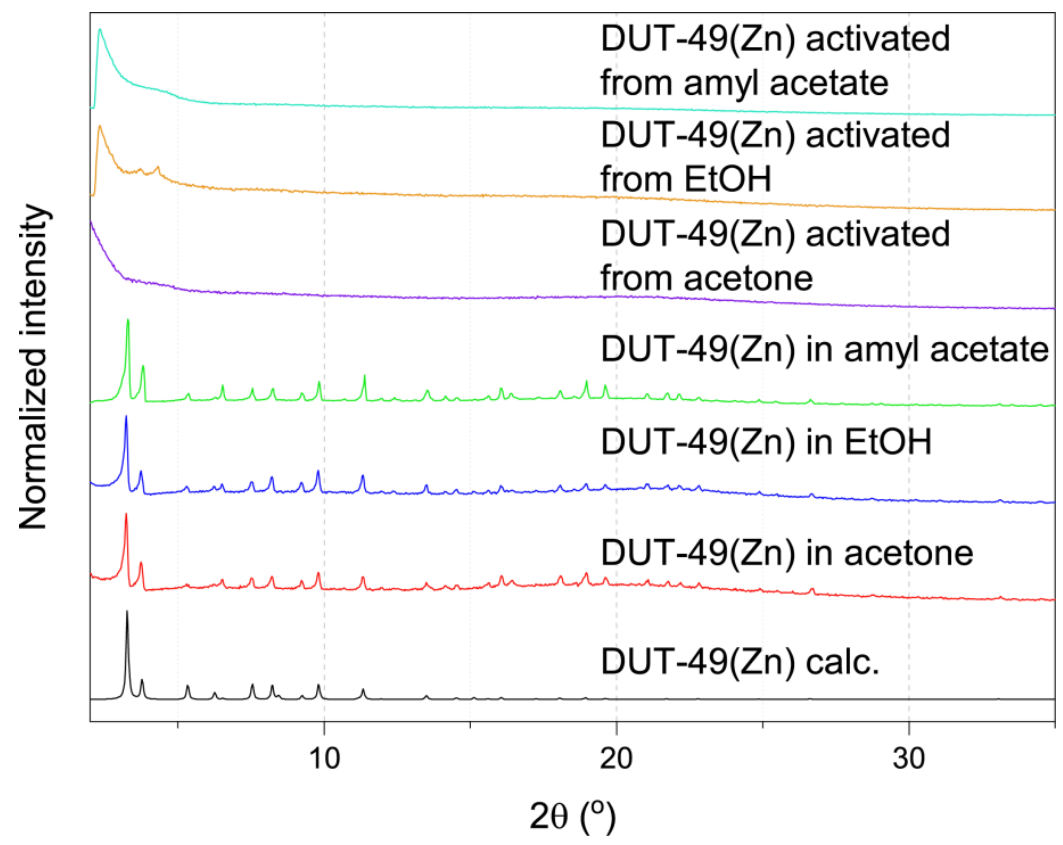

Figure S 7: XRD pattern of DUT-49(Zn) after supercritical $\mathrm{CO}_{2}$ activation from various solvents.

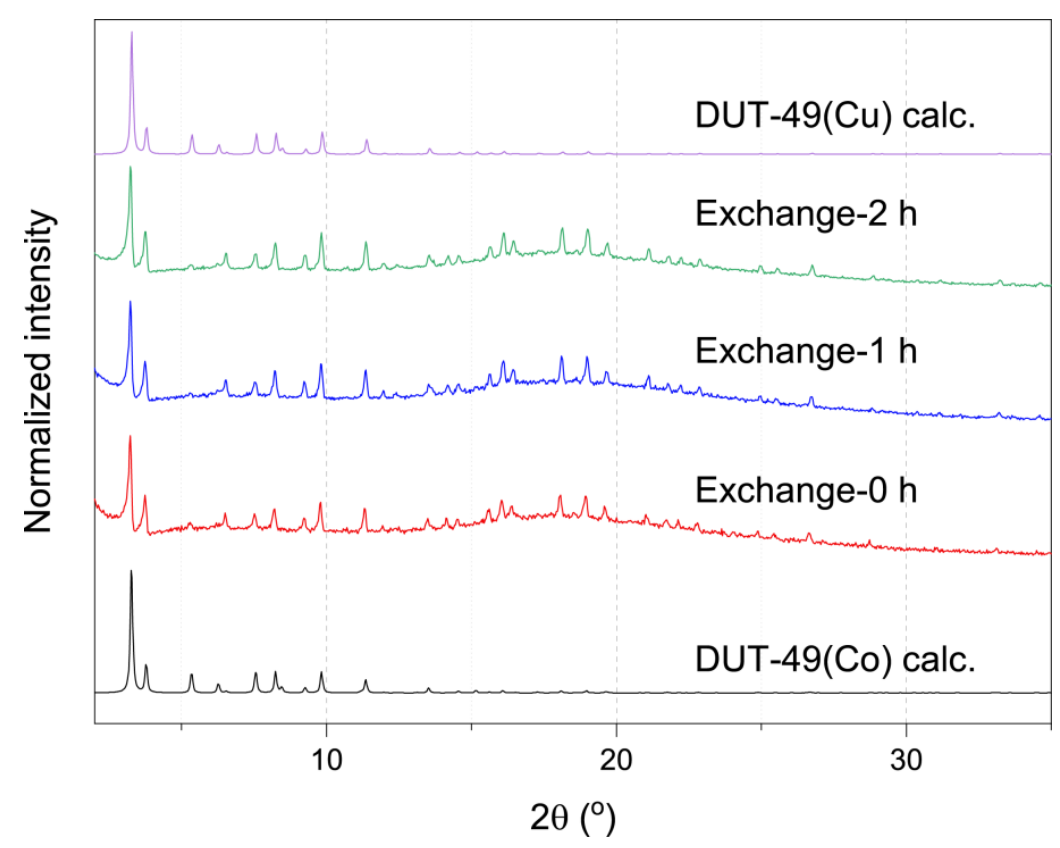

Figure S 8: XRD pattern collected at different time intervals during the conversion of DUT-49(Co) to DUT-49(Cu) in NMP. 


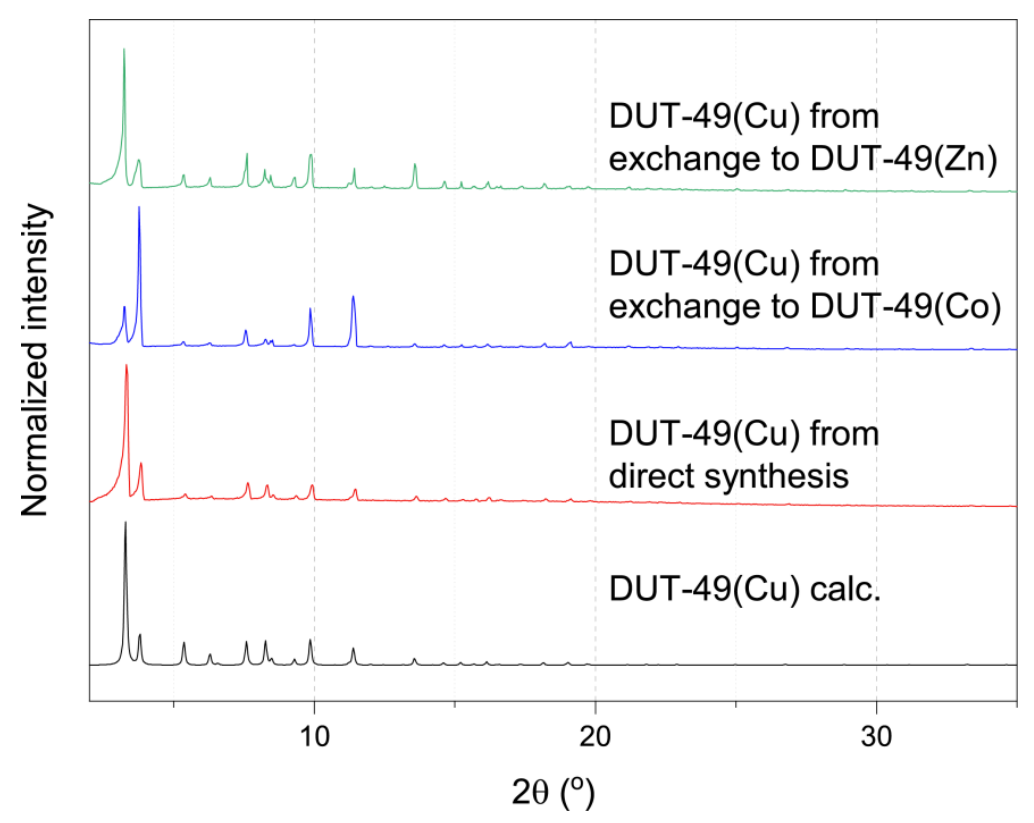

Figure S9: Comparison of XRD pattern for supercritical $\mathrm{CO}_{2}$ activated DUT-49(Cu), obtained from direct synthetic route and from $\mathrm{Cu}$ exchange to DUT-49(Co) and DUT-49(Zn).

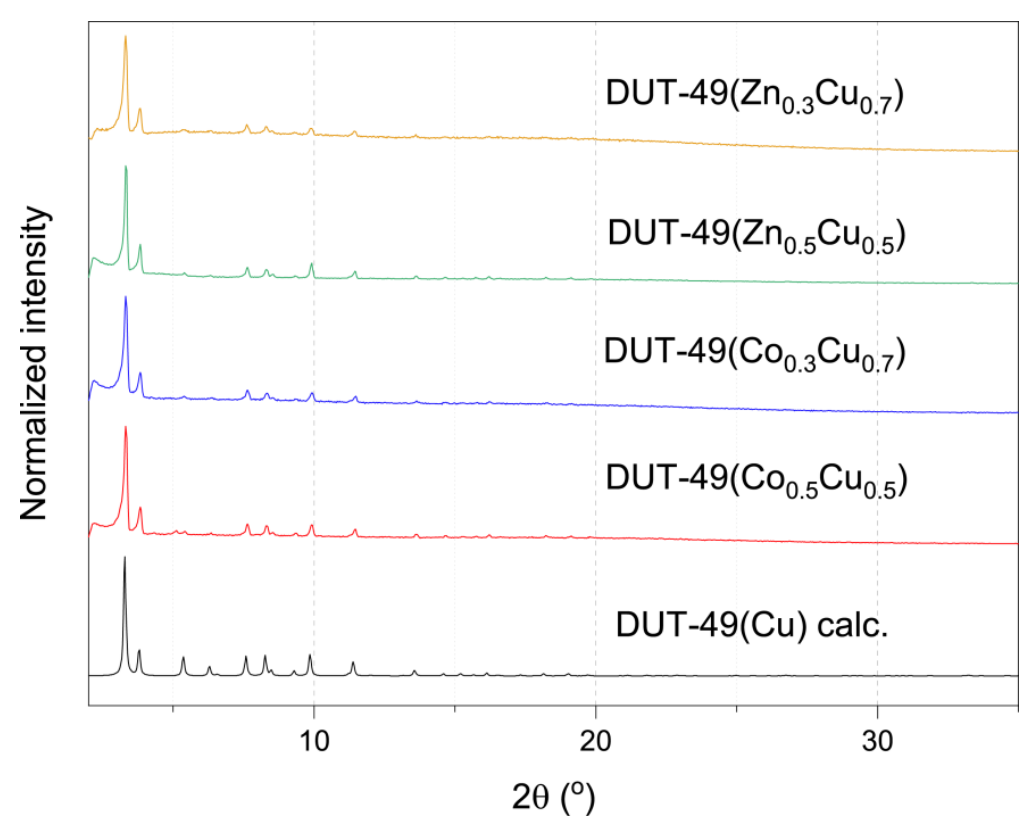

Figure S 10: XRD pattern for supercritical $\mathrm{CO}_{2}$ activated samples having different $\mathrm{Cu}$ content in the DUT-49 framework. 
S7: Porosity and negative gas adsorption of heterometallic DUT-49

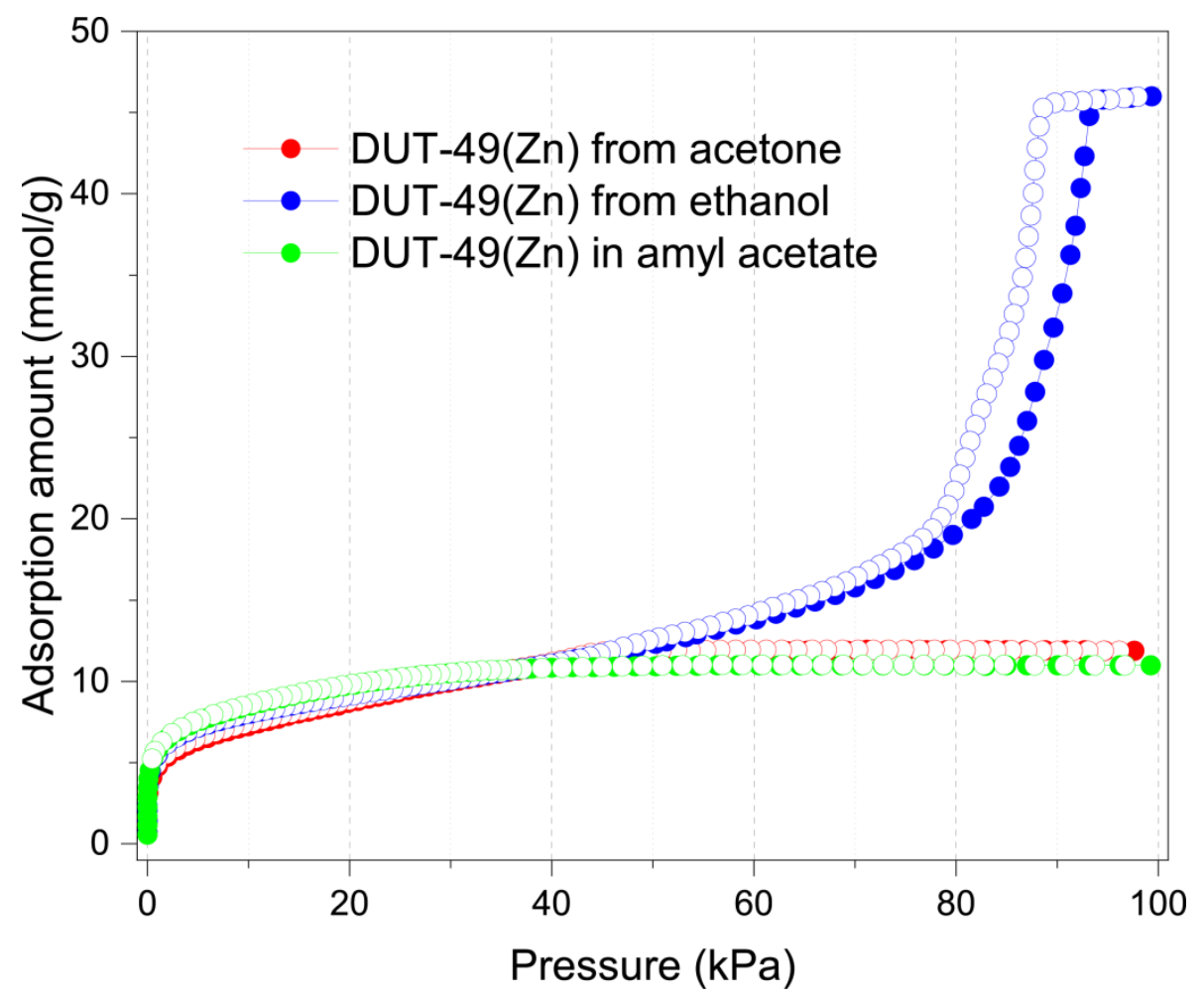

Figure S11: $\mathrm{N}_{2}$ adsorption isotherm for DUT-49(Zn) after SCD activation from different solvents. Filled and empty circles represent adsorption and desorption, respectively.

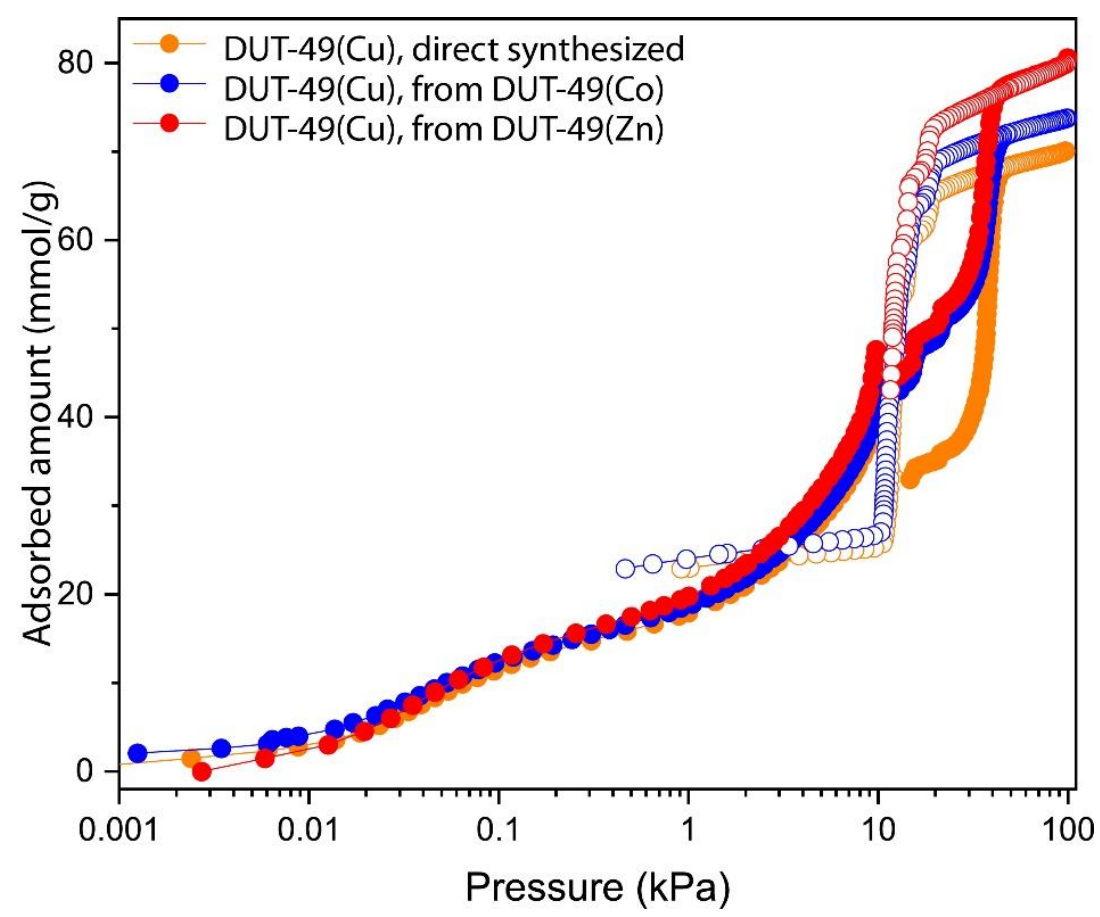

Figure S12: Semi-logarithmic plot of $\mathrm{N}_{2}$ adsorption isotherm of DUT-49(Cu) samples obtained from direct synthesis and metal exchange approaches. Filled and empty circles represent adsorption and desorption, respectively. 


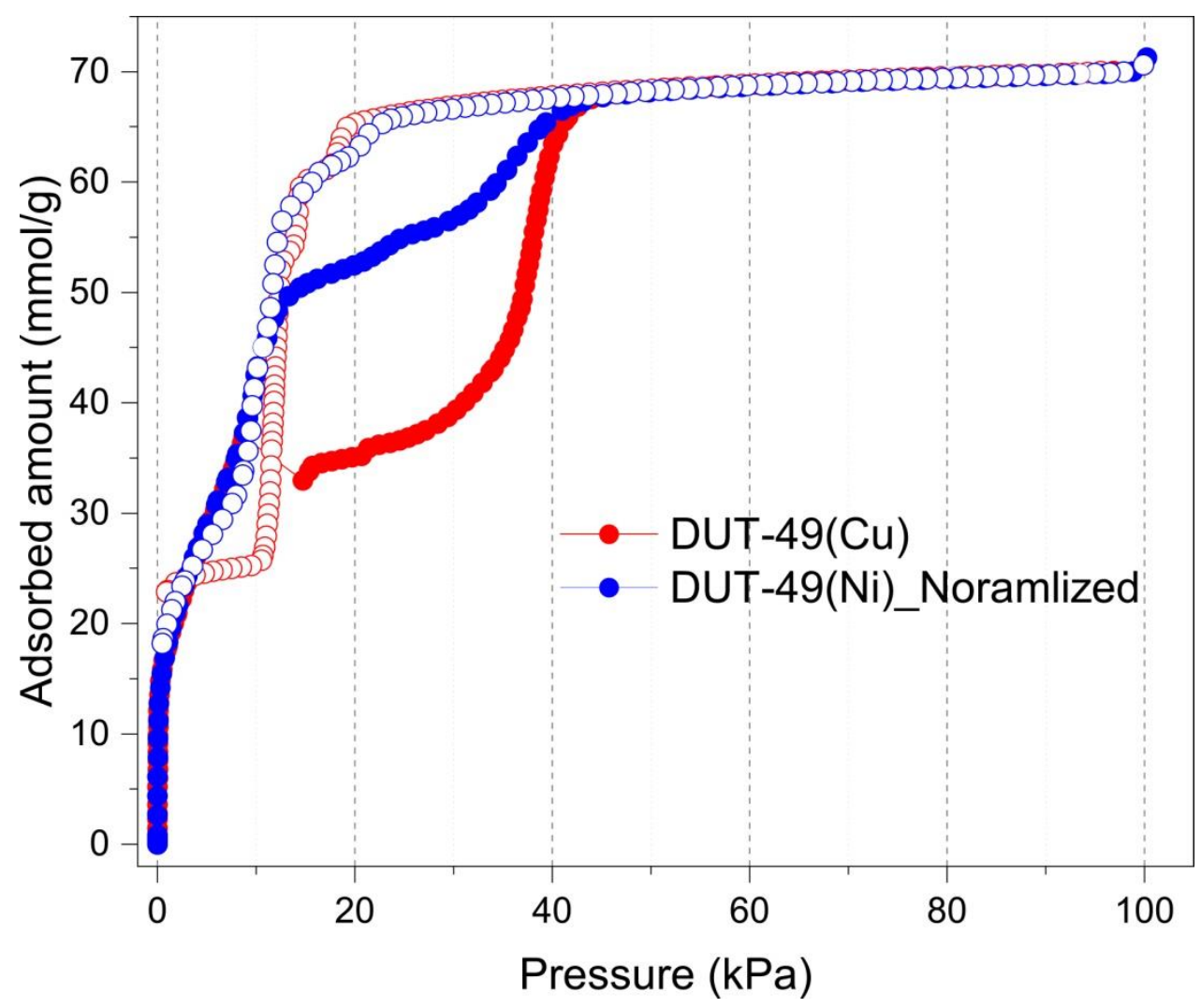

Figure S13: Comparison of $\mathrm{N}_{2}$ adsorption isotherm for DUT-49(Ni), normalized to that for DUT-49(Cu) to show the flexibility of the framework. Filled and empty circles represent adsorption and desorption, respectively.

Table S5: $\mathrm{N}_{2}$ uptake and NGA details for heterometallic DUT-49

\begin{tabular}{|c|c|c|c|}
\hline Sample & $\begin{array}{l}\text { Total Uptake }(\mathrm{mmol} / \mathrm{g}) \\
\text { at } 97 \mathrm{kPa}\end{array}$ & $p_{\mathrm{NGA}}(\mathrm{kPa})$ & $\begin{array}{l}\Delta \mathbf{n}_{\mathrm{NGA}} \\
(\mathrm{mmol} / \mathrm{g})\end{array}$ \\
\hline DUT-49 $\left(\mathrm{Zn}_{1.0} \mathrm{Cu}_{0.0}\right)$ & 11.8 & - & - \\
\hline DUT-49 $\left(\mathrm{Zn}_{0.5} \mathrm{Cu}_{0.5}\right)$ & 23.1 & 90.5 & 1.08 \\
\hline DUT-49 $\left(\mathrm{Zn}_{0.3} \mathrm{Cu}_{0.7}\right)$ & 43.1 & 88.5 & 3.31 \\
\hline DUT-49( $\left.\mathrm{Zn}_{0.0} \mathrm{Cu}_{1.0}\right)$ & 79.8 & 96.2 & 2.87 \\
\hline DUT-49(Cu) & 70.1 & 89.3 & 3.89 \\
\hline
\end{tabular}




\section{S8: SEM imaging and elemental mapping}

Samples for SEM imaging were prepared by spreading activated samples of the MOFs on a conductive sticky carbon tape under argon atmosphere. The sample stage containing the samples in inert atmosphere was then transferred to the microscope and the imaging was carried out under vacuum.
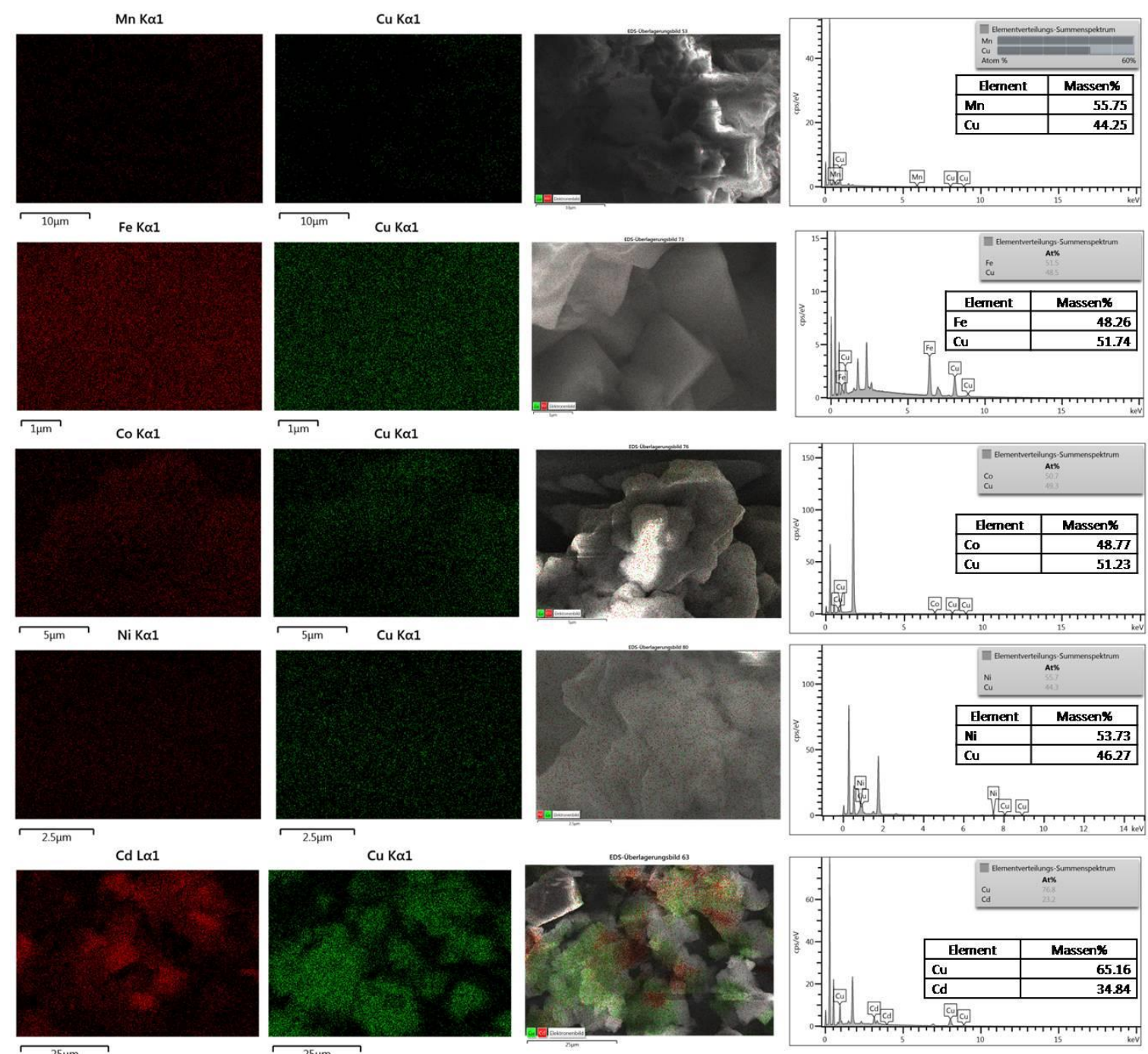

Figure S14: SEM imaging and elemental mapping showing location of different metals on the crystal surface of different heterometallic MOFs. 


\section{S9: References}

1. Stoeck, U.; Krause, S.; Bon, V.; Senkovska, I.;Kaskel, S.A highly porous metal-organic framework, constructed from a cuboctahedral super-molecular building block, with exceptionally high methane uptake. Chem. Commun. 2012, 48, 10841-10843. DOI: 10.1039/C2CC34840C

2. Krause, S.; Bon, V.; Senkovska, I.; Többens, D. M.; Wallacher, D.; Pillai, R. S., Maurin, G.; Kaskel, S. The effect of crystallite size on pressure amplification in switchable porous solids. Nat. Commun. 2018, 9, 1573. DOI: 10.1038/s41467-018-03979-2

3. Helmholtz-Zentrum Berlin für Materialien und Energie. The MX beamlines BL14.1-3 at BESSYII.JLSRF, 2016, 2, 1-6. DOI: 10.17815/jlsrf-2-64

4. Battye, T. G. G., Kontogiannis, L., Johnson, O., Powell, H. R. \& Leslie, A. G. W. iMOSFLM: a new graphical interface for diffraction-image processing with MOSFLM. Acta Crystallogr. D 2011, 67, 271-281. DOI: 10.1107/S0907444910048675

5. Winn, M. D. et al. Overview of the CCP4 suite and current developments. Acta Crystallogr. D 2011, 67, 235-242. DOI: 10.1107/S0907444910045749

6. Sparta, K. M., Krug, M., Heinemann, U., Mueller, U. \&Weiss, M. S. XDSAPP2.0. J. Appl. Crystallogr. 2016, 49, 1085-1092. DOI: $10.1107 /$ S1600576716004416

7. Sheldrick, G. A short history of SHELX. Acta Crystallogr. A 2008, 64, 112-122. DOI: 10.1107/S0108767307043930

8. Sheldrick, G. Crystal structure refinement with SHELXL. Acta Crystallogr. C 2015, 71, 3-8. DOI: $10.1107 /$ S2053229614024218

9. Spek, A. Structure validation in chemical crystallography. Acta Crystallogr. D 2009, 65, 148-155. DOI: $10.1107 /$ S0907444490804362X 